BULL. AUSTRAL. MATH. SOC.

VOL. $20(1979), 165-170$.

\title{
The additive groups of subdirectly irreducible rings
}

\section{Shalom Feigelstock}

An abelian group $G$ is said to be subdirectly irreducible if there exists a subdirectly irreducible ring $R$ with additive group $G$. If $G$ is subdirectly irreducible, and if every ring $R$ with additive group $G$, and $R^{2} \neq 0$, is subdirectly irreducible, then $G$ is said to be strongly subdirectly irreducible. The torsion, and torsion free, subdirectly irreducible and strongly subdirectly irreducible groups are classified completely. Results are also obtained concerning mixed subdirectly irreducible and strongly subdirectly irreducible groups.

1 .

All groups considered here are abelian, with addition the group operation. Although the rings under discussion are not necessarily associative, many of the results remain true for associative rings. The additive group of a ring $R$ will be denoted by $R^{+}$. The torsion part of a group $G$ will be denoted by $G_{t}$. All other notation and terminology will follow [1], [2].

2 .

DEFINITION. A group $G$ is said to be subdirectly irreducible if there exists a subdirectly irreducible ring $R$, with $R^{+}=G$. If every ring $R$ with $R^{+}=G$, and $R^{2} \neq 0$ is subdirectly irreducible, and $G$ is Received 12 January 1979. 
not nil, or if $G$ is nil and the zeroring with additive group $G$ is subdirectly irreducible then $G$ is said to be strongly subdirectly irreducible.

LEMMA 2.1. Let $G$ be a subdirectly irreducible group. Then $G_{t}$ is $p$-primary for some prime $p$.

Proof. If $G_{p}=0$ for every prime $p$, then $G_{t}=0$. Otherwise let $p$ be a prime for which $G_{p} \neq 0$. Let $R$ be a subdirectly irreducible ring. For every $q \neq p, G_{p} \cap G_{q}=0$. Since $G_{p}$ and $G_{q}$ are ideals in $R, G_{q}=0$.

THEOREM 2.2. Let $G$ be a torsion group. $G$ is subdirectly irreducible if and only if $G$ is a bounded p-primary group, or if $G$ is a p-primary group with first ULm subgroup $G^{\prime} \simeq Z\left(p^{k}\right), \quad 1 \leq k \leq \infty$.

Proof. Suppose that $G$ is subdirectly irreducible. By Lemma 2.1, $G$ is $p$-primary for some prime $p$. Let $R$ be a subdirectly irreducible ring with $R^{+}=G$. Suppose that $G^{\prime}=0$. Then $\prod_{n<\omega} p^{n} G=0$. However $p^{n} G$ is an ideal in $R$ for every positive integer $n$, and so $p^{n} G=0$ for some $n<\omega$; that is, $G$ is bounded.

Suppose that $G^{\prime} \neq 0$. Let $H$ be a subgroup of $G^{\prime}$. Clearly $R H=H R=0$. Hence $H$ is an ideal in $R$. Therefore, if $G^{\prime}=H \oplus K$, then $H$ and $K$ are ideals in $R$ with trivial intersection; that is, $G^{\prime}$ is indecomposable. Hence $G^{\prime} \simeq 2\left(p^{k}\right), I \leq k \leq \infty \quad[1$, Corollary 27.4].

Let $G$ be a bounded $p$-primary group with basis $\left\{a_{i} \mid i \in I\right\}$, $\left|a_{i}\right|=p^{k_{i}}, k_{i} \leq k$ for all $i \in I$. Choose $i_{0} \in I$ such that $k_{i_{0}}=k$. For $i, j \in I$ define

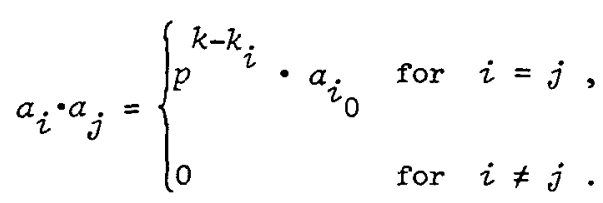

This composition induces a ring structure $R$ on $G$ [2, Theorem 120.1]. 
Let $x \in R, x \neq 0$. Then $x=n_{1} a_{i_{1}}+\ldots+n_{r} a_{i_{r}},{ }^{i} j$ distinct elements of $I$, and $n_{j}$ an integer with $p^{k_{i}} h_{n_{j}}$ for $j=1, \ldots, r$. Now $x a_{i_{1}}=n_{1} p^{k-k_{1}} \cdot a_{i_{0}}$. Therefore every nonzero ideal in $R$ contains $p^{k-1} a_{i_{0}}$, and so $R$ is subdirectly irreducible.

Let $G$ be a $p$-primary group, with $G^{\prime} \simeq z\left(p^{k}\right), 1 \leq k \leq \infty$. Then $G^{\prime}$ possesses a unique minimal subgroup generated by some $a_{0} \in G$, $\left|a_{0}\right|=p$. For every positive integer $k$, choose $a_{k} \in G$ such that $p^{k} a_{k}=a_{0}$. Let $B$ be a basic subgroup of $G$ with basis $\left\{b_{i} \mid i \in I\right\}$, $\left|b_{i}\right|=p^{k_{i}}$ for all $i \in I$. For $i, j \in I$ define

$$
b_{i} \cdot b_{j}= \begin{cases}a_{k_{i}-1} & \text { for } i=j, \\ 0 & \text { for } i \neq j .\end{cases}
$$

This composition induces a ring structure $R$ on $G$ [2, Theorem 120.1]. Let $a \in R, \quad a \neq 0,|a|=p^{j}$. Now $B=\underset{n<\omega}{\oplus} B_{n}$, with $B_{n} \simeq \underset{\alpha_{n}}{\oplus} z\left(p^{n}\right)$, $\alpha_{n}$ a cardinal number, and $B_{n}$ a direct summand of $G$ for all $n<\omega$. Let $r$ be the smallest positive integer such that under the projection $\pi_{r}: G \rightarrow B_{r}, \pi_{p}(a) \neq 0$. Let $n$ be a positive integer, $n \geq j+r$.

Since

$$
G=\bigoplus_{1 \leq i \leq n}^{\oplus} B_{i} \underset{n+1 \leq i<\omega}{\oplus} B_{i}+p^{n} G
$$

$a=m_{1} b_{i_{1}}+\ldots+m_{s} b_{i_{s}}+g$, with $i_{t}$ distinct elements of $I, m_{t}$ an integer, $t=1, \ldots, s, k_{i_{1}}=r, m_{1}=p^{u} \cdot v, u, v$ integers, $u<r,(p, v)=1$, and $g \in p^{r} G$. Now $a \cdot p^{r-u-1_{b}}{ }_{i_{1}}=v a_{0}$. Therefore 
every nonzero ideal of $R$ contains $a_{0}$.

OBSERVATION 2.3. Let $G$ be a mixed subdirectly irreducible group. Then $G_{t}$ is a $p$-primary group possessing nonzero elements of infinite height.

Proof. $G_{t}$ is $p$-primary by Lemma 2.1. Let $R$ be a subdirectly irreducible ring, with $R^{+}=G$. For every positive integer $n, p^{n_{G}}$ is an ideal in $R$. If $\bigcap_{n<\omega} p^{n} G_{t} \neq 0$, then $G$ possesses nonzero elements of infinite $\quad$ Otherwise, $p^{n} G_{t}=0$ for some positive integer $n$. In the latter case $G_{t}$ and $p^{n} G$ are nonzero ideals in $R$ satisfying $G_{t} \cap p^{n} G=0$, a contradiction.

THEOREM 2.4. Let $G$ be a torsion free group. $G$ is subdirectly if and only if $G$ is not reduced.

Proof. Suppose that $G$ is subdirectly irreducible. Let $R$ be a subdirectly irreducible ring with $R^{+}=G$. The ath Ulm subgroup $G^{\alpha}$ of $G$ is an ideal in $R$ for every ordinal $\alpha$. Let $\alpha$ be the smallest ordinal for which $G^{\alpha}=G^{\alpha+1}$. If $\alpha$ is a limit ordinal, then $G^{\alpha}=\bigcap_{\beta<\alpha} G^{\beta}$ is the intersection of nonzero ideals in $R$, and so $G^{\alpha} \neq 0$. If $\alpha$ is an isolated ordinal, say $\alpha=\beta+1$, then $G^{\alpha}=n_{n<\omega} n G^{\beta}$ is the intersection of nonzero ideals $n G^{\beta}$ of $R$, and so $G^{\alpha} \neq 0$. Therefore $G^{\alpha}$ is a nonzero, divisible subgroup of $G$.

Suppose that $G$ is not reduced. Then $G=D \oplus H, D$ a divisible group, $H$ a reduced group, $D \neq 0$. Let $\left\{e_{i} \mid i \in I\right\}$ be a maximal independent set for $D$, and let $\left\{a_{j} \mid j \in J\right\}$ be a maximal independent set for $H$. Choose $i_{0} \in I$. Define 


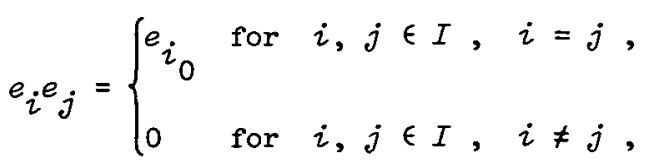

$$
\begin{aligned}
& e_{i} a_{j}=\alpha_{j} e_{i}=0 \text { for } i \in I, j \in J \text {, }
\end{aligned}
$$

and

$$
a_{i} a_{j}= \begin{cases}e_{i} & \text { for } i, j \in J, i=j, \\ 0 & \text { for } i, j \in J, i \neq j .\end{cases}
$$

This composition defines a ring structure $R$ on $G$. Every nonzero ideal in $R$ contains $e_{i_{0}}$, and so $R$ is subdirectly irreducible.

3.

LEMMA 3.1. Let $G$ be a strongly subdirectly irreducible group, $G=H \oplus K, \quad H \neq 0$, and $K \neq 0$. Then $H$ and $K$ are both niz.

Proof. Suppose that $H$ is not nil. Let $S$ be a ring with $S^{+}=H$, $S^{2} \neq 0$, and let $T$ be a ring with $T^{+}=K$. The ring direct sum $R=S \oplus T$ has additive group $G, R^{2} \neq 0$, and $R$ has nonzero ideals $S, T$ with $S \cap T=0$, a contradiction.

THEOREM 3.2. Let $G$ be a torsion free group. $G$ is strongly subdirectly irreducible if and only if $G \simeq Q$.

Proof. By Theorem 2.4, $G \simeq Q \oplus H$. Hence $G \simeq Q$ by Lenma 3.1. The converse is obvious.

THEOREM 3.3. Let $G$ be a mixed strongly subdirectly irreducible group. Then $G \simeq 2\left(p^{\infty}\right) \oplus H, H$ a rank one, torsion free nil group.

Proof. By [1, Corollary 27.3], $G \simeq 2\left(p^{k}\right) \oplus H, 1 \leq k \leq \infty$. Hence by Lemma 3.1 and [2, Theorem 120.3], $k=\infty$, and $H$ is a nil torsion free group.

Suppose that $r(H)=\alpha>1$. Then $r(H \otimes H)=\alpha^{2}>1 \quad[1$, Exercise 8 , p. 261]. Let $D$ be the divisible hull of $H \otimes H$. Then $D=Q_{1} \oplus K$, $Q_{1} \simeq Q, K \neq 0$. Let $x \in Q_{1}, x \neq 0$. There exists a positive integer 
$m$ such that $m x \in H \otimes H$. Now $m=p^{k} n,(p, n)=1$. Let $a \in Z\left(p^{\infty}\right)$, $|a|>p^{k}$. There exists a nonzero homomorphism $\phi: Q_{1} \rightarrow Z\left(p^{\infty}\right)$ such that $\phi(x)=a$. Now $\phi(m x)=m \phi(x) \neq 0$. Let $\pi$ be the projection of $D$ onto $Q_{1}$ and put $\psi=\phi \pi$. Then $\psi_{H}$ the restriction of $\psi$ to $H \otimes H$ is a nonzero homomorphism. Let $g_{i}=a_{i}+b_{i}, a_{i} \in Z\left(p^{\infty}\right), b_{i} \in H$, $i=1,2$. Define $g_{1} * g_{2}=\psi_{H}\left(b_{1} \otimes b_{2}\right)$. Then $R=(G, *)$ is a ring with $R^{+}=G$, and $R^{2} \neq 0$. Let $h \in H$ be such that $h \otimes h \in(H \otimes H) \cap K$. Let $L$ be the subgroup of $H$ generated by $h$. Then $Z\left(p^{\infty}\right)$ and $L$ are ideals in $R$, with $Z\left(p^{\infty}\right) \cap L=0$.

THEOREM 3.4. Let $G$ be a torsion group. $G$ is strongly subdirectly irreducible if and only if $G \simeq 2\left(p^{k}\right), \quad 1 \leq k \leq \infty$.

Proof. Suppose that $G$ is strongly subdirectly irreducible. By [1, Corollary 27.3], $G \simeq Z\left(p^{k}\right) \oplus H, I \leq k \leq \infty$. If $k<\infty$, then $H=0$ by Lemma 3.1. If $k=\infty$ then $H$ is nil by Lemma 3.1 , and hence divisible [2, Theorem 120.3]. $G$ is nil. $Z\left(p^{\infty}\right)$ and $H$ are ideals in the zeroring with additive group $G$, and $Z\left(p^{\infty}\right) \cap H=0$. Therefore $H=0$.

The converse is obvious.

\section{References}

[1] László Fuchs, Infinite abelian groups, Volume I (Pure and Applied Mathematics, 36. Academic Press, New York and London, 1970).

[2] László Fuchs, Infinite abelian groups, Volume II (Pure and Applied Mathematics, 36-II. Academic Press, New York and London, 1973).

Department of Mathematics,

Bar-llan University,

Ramat-Gan,

Israel. 